\title{
Spontaneous nervous system concussion in dogs: a description of two cases and a review of terminology in veterinary medicine
}

\author{
Angelo Pasquale Giannuzzi, Antonio De Simone and Mario Ricciardi* \\ “Pingry” Veterinary Hospital, via Medaglie d'Oro 5, Bari, Italy
}

\begin{abstract}
In human medicine, central nervous system (CNS) concussion is defined as a transient neurological dysfunction following a traumatic event, without evidence of structural abnormalities of the affected region on advanced diagnostic imaging. Depending on the anatomical region involved, three forms of concussive syndromes are described: brain concussion, spinal concussion and cerebellar concussion. Although major textbooks of veterinary neurology admit the existence of canine brain concussion, spontaneous cases of this pathological condition have not been reported in small animals so far. This report describes two cases of concussion in dogs: a 9-month-old, intact male, shih-tzu with brain concussion; and a 10-month-old, intact male, poodle with cerebellar concussion. In addition, a brief review of the definition of the term "concussion" in the veterinary medical literature is provided, in comparison to its meaning in the human medical literature. Finally, this paper proposes an appropriate definition of "concussion" in dogs, that may facilitate clinicians in the recognition of such an elusive syndrome.
\end{abstract}

Keywords: Brain, Computed tomography, Concussion, Dog, Magnetic resonance imaging.

\section{Introduction}

Concussion of the central nervous system (CNS) is characterized by rapid and complete resolution of neurological signs following a traumatic event without evidence of structural lesions of the affected anatomical regions on advanced diagnostic imaging (Snedden, 2013). Depending on the area involved, three types of CNS concussion are reported in human patients: brain concussion, spinal cord concussion and cerebellar concussion (Zwimpfer and Bernstein, 1990; Fumeya and Hideshima, 1994). Although transitory, clinical signs may be severe and related to the CNS area involved in the concussive event. Temporary unconsciousness, abolition of various reflex functions, amnesia and cerebellar dysfunction are described in human patients with head concussion, while a wide range of spinal cord dysfunction, ranging from paresthesia to quadriplegia, is reported for spinal concussion (Fumeya and Hideshima, 1994; Winder et al., 2011; Snedden, 2013).

To date, although major textbooks of veterinary neurology (Platt et al., 2001; Lorenz et al., 2011; Platt and Garosi, 2012) admit the existence of canine brain concussion, no cases of such a pathological condition have been documented in dogs. Furthermore, canine cerebellar concussion, other than spinal concussion, has never been reported before.

On the other hand, when the term concussion is used in veterinary medicine, it is more often linked to spinal cord injury with structural damage that is detectable on diagnostic imaging and is often adopted, confusingly, as a synonym for contusion (Olby, 1999; Ferreira et al., 2002; Kube and Olby, 2008).

Although several experimental studies on CNS concussion have been performed in small animals (Denny-Brown and Russell, 1940; Scott, 1940; Walker et al., 1944), clinical syndromes related to spontaneous concussive damage of the nervous system have not been described in dogs so far.

This report describes two cases of presumptive CNS concussion in dogs and discusses the neurophysiopathologic basis of such a condition. Moreover, a brief review of the terminology adopted in veterinary medicine, in comparison with the human medical literature, is provided.

\section{Case Details}

$\operatorname{Dog} 1$

A nine-month-old, intact male, shih-tzu dog was referred for a sudden loss of consciousness that occurred during the washing process in a dog-wash store six hours before clinical evaluation. A witness present during the accident reported that the dog was frightened by a hair dryer, and in an attempt to get out from his collar he made vigorous jolting movements with his head and then collapsed as if he was dead. A few minutes later the dog regained some awareness, but was unable to stand and appeared poorly responsive to environmental stimuli.

Physical and neurological examination revealed bradycardia (60 bpm), lateral recumbency and severe depression. If the patient was manually sustained, flaccid tetraplegia and neck ventroflexion were evident. 
Withdrawal reflexes were absent in all four limbs as were the patellar reflexes on both sides. Menace reaction was bilaterally absent and there was bilateral nasal analgesia along with a loss of nociception in all limbs (Fig. 1-A, B).

Based on neurological examination a multifocal neurological syndrome was suspected. $\mathrm{CBC}$, biochemical profile and urinalysis were also performed, to rule out concomitant diseases.

In order to clearly evaluate possible skeletal injuries of the skull and cervical vertebrae, an unenhanced CT scan of the cranio-cervical region was acquired using a four slice-MDCT scanner (Philips MX-8000*) with the following technical parameters: bone and spine acquisition algorithm, $120 \mathrm{kVp}, 200 \mathrm{mAs}, 1.5 \mathrm{~mm}$ slice thickness, pitch of 0.8 , and 0.75 s/rotation. Threedimensional (3D) multiplanar reformatted and volumerendered images were obtained using a dedicated 3D workstation (OsiriX DICOM-viewer: Pixmeo, Geneva, Switzerland).

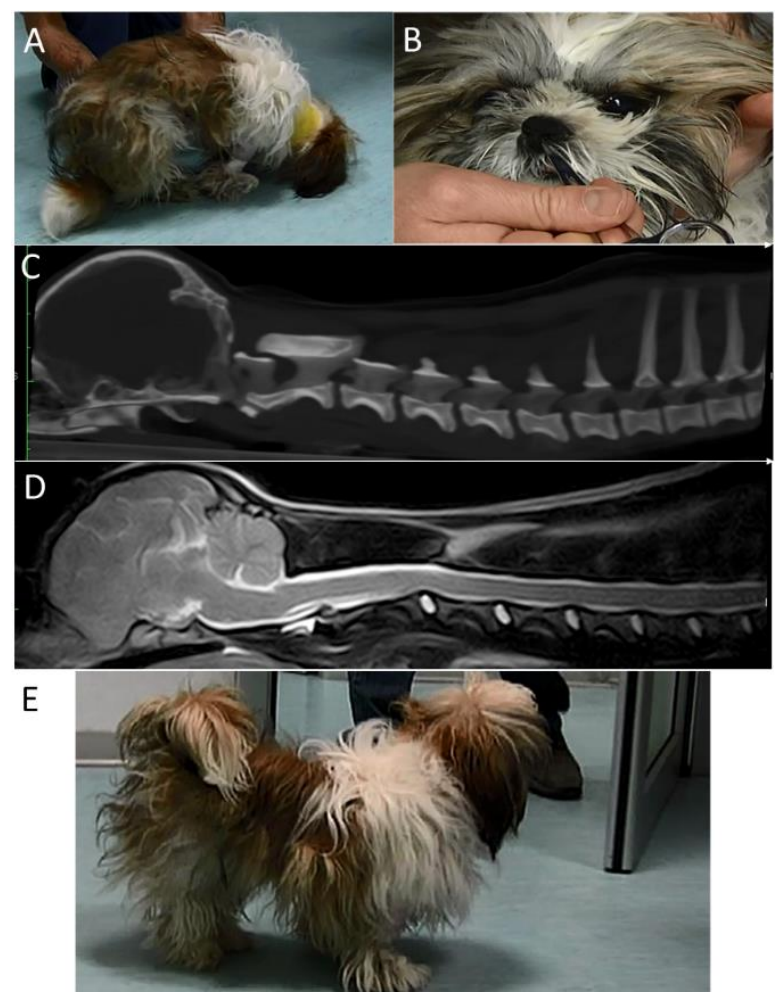

Fig 1. Dog 1: Nine-month-old, intact male, Shih-Tzu dog. (A, B): Frames extracted from a movie of the neurological examination showing: (A): flaccid tetraparesis with neck ventroflexion and (B): nasal analgesia. (C): Midsagittal thick-slab multiplanar reformatted CT image of the head and cervical spine showing the absence of bone lesions on the calvarium and cervical vertebrae. (D): Midsagittal T2weighted MRI image of the head and cervical spine showing no structural or signal abnormalities of the brain or spinal cord. (E): Thirty-six hours after traumatic event the dog fully recovered from his neurological condition.
In addition, brain and spinal cord parenchyma were evaluated by cranio-cervical MRI, using a 0.25 Tesla permanent magnet (ESAOTE VET-MR GRANDE, Esaote, Genoa, Italy) with the following sequence protocol: Fast Spin Echo T2-weighted images acquired in sagittal and transverse plane, FLAIR and STIR acquired in the transverse plane and Spin Echo T1weighted images acquired in the transverse plane, before and after intravenous administration of paramagnetic contrast medium (Magnegita gadopentatedimeglumine $500 \mathrm{mmol} / \mathrm{mL}$ - insight agents; $0.15 \mathrm{mmol} / \mathrm{kg} \mathrm{BW).} \mathrm{CT} \mathrm{and} \mathrm{MRI} \mathrm{images} \mathrm{were}$ carefully evaluated consensually by both authors.

On CT images, no bone lesions were found in the skull or cervical vertebrae (Fig 1-C). No structural lesions or signal changes were seen in the brain or spinal cord in MRI images (Fig 1-D).

The patient underwent cage rest, fluid therapy and clinical observation. Thirty-six hours after the trauma the dog completely recovered from his neurological condition and was discharged (Fig 1-E).

\section{$\operatorname{Dog} 2$}

A 10-month-old, intact male, poodle dog was evaluated for head trauma that occurred two hours before examination. The owner reported that the dog fell backwards from a table onto the ground and suffered a blunt trauma to the nape of its neck. Neurological examination revealed normal mental status, head intentional tremor and truncal ataxia. During ambulation, the patient fell on both sides. If manually sustained, hypermetria, most evident in both forelimbs was seen. Positional proprioception was absent in all four limbs and spinal reflexes were all normal with increased muscle tone in the forelimbs. Bilateral loss of menace reaction was also detected. Overall, the neurological findings were suggestive of diffuse cerebellar dysfunction.

Brain MRI and CT were carried out, with the same technical parameters as described for $\operatorname{dog} 1$, to rule out any possible underlying traumatic cerebellar lesion. CT and MRI images were carefully evaluated consensually by both authors and no structural abnormalities were detected using either technique (Fig 2).

The patient was hospitalized for clinical observation and treated with fluid therapy. Twenty-four hours later the dog was able to walk and run, and only a mild hypermetria was present in the forelimbs. Based on the owner's opinion the dog was back to normal three days after discharge.

\section{Discussion}

The term "concussion" comes from the latin word "concutere" which means "to shake violently". From a biomechanical point of view, brain concussion is typically induced by a sudden acceleration or deceleration of the head (Denny-Brown and Russell, 1940; Bannister, 1992; Rosenthal, 1993; Label, 1997). 

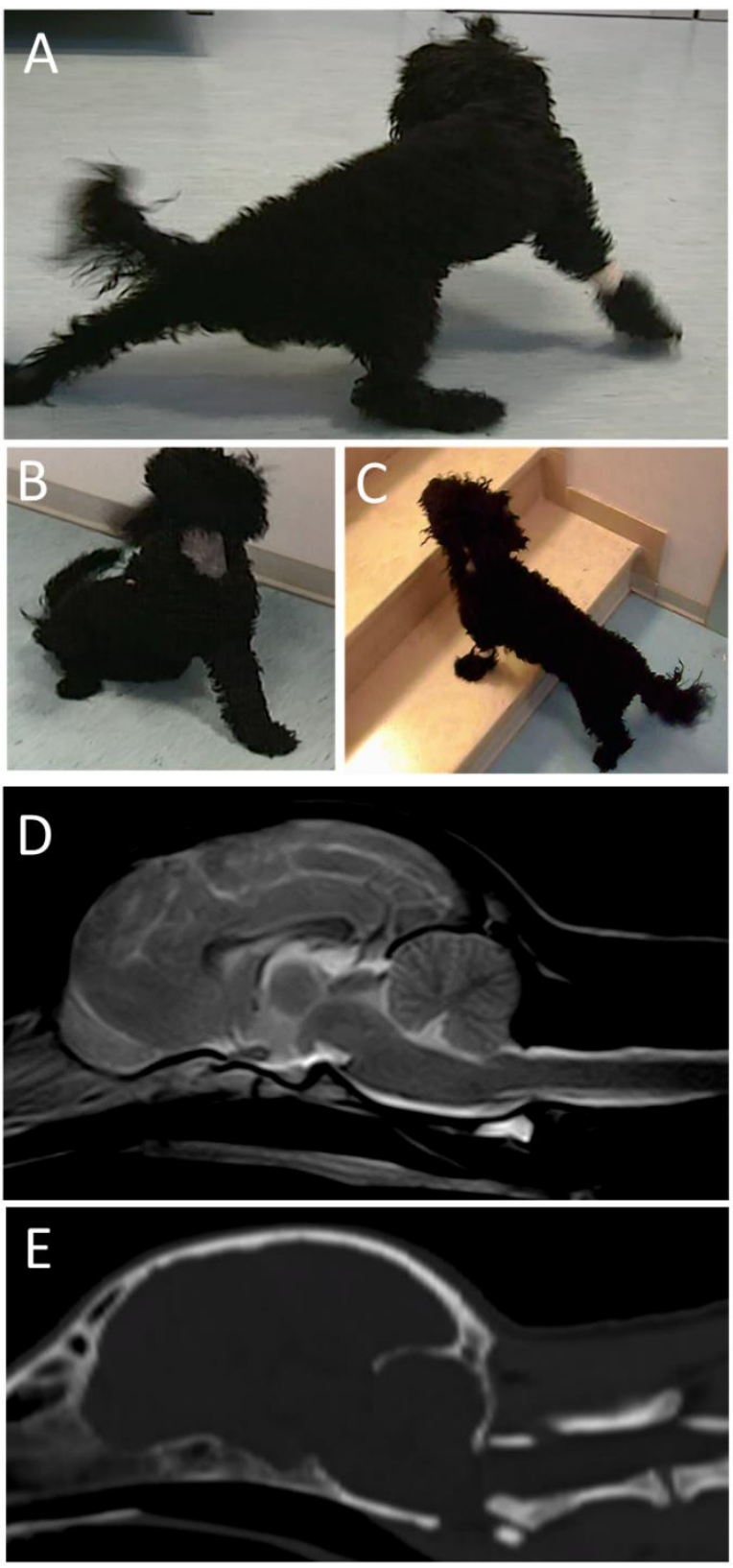

Fig 2. Dog 2: Ten-month-old, intact male, poodle dog. (A, B, C): Frames extracted from a movie of the neurological examination showing: (A, B): cerebellar ataxia and (C): twenty-four hours after the traumatic event the patient was able to walk. (D): Midsagittal T2-weighted MRI image of the head showing no structural or signal abnormalities of the brain. (E): Midsagittal multiplanar reformatted CT image of the head showing the absence of bone lesions on the calvarium.

Clinical presentation of temporary unconsciousness, abolition of various reflex functions and amnesia are the main clinical signs and symptoms of brain concussion (Snedden, 2013). Loss of consciousness is not considered a constant feature of cerebral concussion, occurring only in $10 \%$ of cases (Snedden, 2013).

Although definition of brain concussion is a matter of dispute, when transient loss of consciousness occurs, associated with an absence of structural lesions in advanced diagnostic images, a diagnosis of concussive trauma is indubitable (Snedden, 2013).

Although brain concussion is generally considered a harmless pathological condition, three types of neurological consequences are suspected to be associated with this injury: post-concussion syndrome, second impact syndrome and chronic traumatic encephalopathy.

Prolonged recovery from concussion symptoms taking longer than seven days is considered to be postconcussion syndrome (Larrabee, 1997; Satz et al., 1999). Although rare, there are reports where postconcussion symptoms take as long as five years to clear up after trauma (Cantu et al., 2010). Second impact syndrome is the most concerning concussion-related problem. This is a rare, often fatal, traumatic brain injury that may occur when a second brain trauma occurs before the symptoms of the first trauma have been resolved (Cantu, 1998). Typically, the second head injury is only a minor blow to the head, but can lead to coma cause by cerebral edema, and brain herniation (McKee, 2014). Chronic traumatic encephalopathy is a neurodegenerative disease caused by repetitive head impacts, which can also occur without any reported or known symptomatic concussions (Stern et al., 2011; Blennow et al., 2012). Those at highest risk are professional athletes who participate in contact sports (mainly boxers) and military personnel who are exposed to repetitive blast events (Petrie et al., 2014).

Symptoms may begin years after repetitive brain trauma exposure and include memory loss and other cognitive impairments (Stern et al., 2013; Montenigro et al., 2015a). The diagnosis is made postmortem and is defined by the presence of $\mathrm{p}$-tau protein in the form of neurofibrillary tangles, glial tangles and neuropil threads, with widespread distribution throughout the brain (Montenigro et al., 2015a,b; McKee et al., 2015). Recently, a suspected chronic traumatic brain injury was documented in a dog submitted to repeated aggression and physical abuse by its owner (Laurent $e t$ al., 2010).

Spinal cord concussion is characterized as a fully reversible condition caused by trauma, with neurological signs associated with spinal cord injury, ranging from paresthesia to quadriplegia, without signs of structural changes (Winder et al., 2011). All patients experience rapid resolution of the neurological deficit within 72 hours of injury (Nesnidal et al., 2012). The most frequent presentation of spinal concussion is due 
to cervical spinal injury, and it is also named cervical cord neuropraxia (Torg et al., 1986).

Finally, cerebellar concussion in people is a rare type of brain injury characterized by transient cerebellar dysfunction following head trauma, with normal findings on computed tomography examination. These findings distinguish cerebellar concussion form cerebellar contusion (Fumeya and Hideshima, 1994). Analyzing the term "concussion" in human medical literature, irrespective of whether the brain, cerebellum or spinal cord is involved, it is characterized by three accepted features: 1) it is always caused by trauma and in some circumstances it has a specific biomechanical dynamic; 2) neurological signs are typically transient and full recovery almost always occur without medical or surgical treatment; 3) diagnostic imaging (radiographs, MRI, CT) shows normal findings or lesions may eventually be detected that are not proportional to the clinical syndrome (McCrory et al., 2013; Snedden, 2013).

The neurophysiology of brain concussion is not completely understood (Snorf and Lynch, 1957; McCrory and Berkovic, 2001; Shaw, 2002). Although it is well accepted that structural damage may occur, nevertheless, the clinical features of concussion primarily reflect a functional neuronal disturbance (McCrory and Berkovic, 2001).

The first attempt to explain concussion was the vascular hypothesis (Trotter, 1924; Scott, 1940). Its chief tenet is that the loss of consciousness and other functions following concussion are due to a brief episode of cerebral ischemia. Vasospasm or vasoparalysis, reflex stimulation, expulsion of the blood from the capillaries and, most commonly, obstruction or arrest of CBF following compression of the brain are hypothesized as mechanisms that could trigger this ischemic event (Shaw, 2002).

More recent hypotheses have focused attention on brainstem dysfunction in order to explain signs and symptoms of brain concussion (Windle et al., 1944; Ommaya and Gennarelli, 1974; Gordon and Ponten, 1976; Hayes et al., 1986). Brainstem dysfunction is suspected to occur through direct or indirect mechanisms.

According to the reticular theory, ascending reticular formations in the brainstem would be primarily involved in concussive trauma (Windle et al., 1944; Gordon and Ponten, 1976). Sudden rotation of the cerebrum within the cranial vault, or rapid flexion or extension of the brainstem on the axis of the cervicomedullary junction may create shearing among brain stem pathways. Hence, brain stem dysfunction would be at the basis of the signs and symptoms of concussive syndrome.

Regarding the pathophysiological changes in ascending reticular activating system (ARAS) which might be triggered by a concussive blow, one of the most enduring theories involves a role for acetylcholine. It has long been reported that acetylcholine may be abnormally abundant in the CSF following both clinical and experimental head injury (Bornstein, 1946; Tower and McEachern, 1948; Sachs, 1957). Accordingly, sudden deformation or compression of the brainstem might release copious amounts of acetylcholine, which flood the intercellular space and temporarily impede synaptic transmission within the ARAS (Hayes et al., 1986).

Indirect involvement of the brainstem has been hypothesized in the centripetal theory (Ommaya and Gennarelli, 1974). In this case, the principal site of action of concussion must lie, not deep within the brainstem, but rather superficially at the cortex. Thus, cerebral concussion would be caused by mechanically induced strains affecting the brain in a centripetal sequence, with destructive effect. This sequence always begins at the surfaces of the brain and extend inwards to affect the diencephalic-mesencephalic core. Disconnection of the brainstem will disrupt the function of the rostral ARAS as well as paralyzing motor performance.

Finally, the convulsive theory is based on the assumption that concussion initially excites rather than temporarily depresses cellular function (Walker et al., 1944). Therefore, concussion is conceived as a kind of epileptic seizure.

In this case, the brain's immediate response to a concussive blow seems to be a hyperexcitatory state due to widespread neuronal membrane depolarization. Neuronal exhaustion, inhibition or extinction would account for the subsequent longer and more salient post-ictal period of paralysis, stupor and depressed cortical rhythms.

With respect to cerebellar concussion, little is known about its pathophysiology due to the scarce documentation in the medical literature (Cantu, 1969; Kanios, 1981; Kepski, 1983; Fumeya and Hideshima, 1994; Gohil and Munshi, 2006; Yeoh et al., 2006). It has been suggested that cerebellar concussion may be due to reversible ischemia of some cerebellar components from post-traumatic vasospasm (Fumeya and Hideshima, 1994).

A common feature of the two cases described in this paper is the observation of a transient traumatic neurological syndrome. In addition, advanced diagnostic imaging in both dogs did not show any abnormality, ruling out structural lesions related to traumatic events or concomitant diseases. Thus, the history, clinical and diagnostic findings fully respect the diagnostic criteria of nervous system concussion as it is described in humans.

In $\operatorname{dog} 1$, based on the reversible loss of consciousness, flaccid tetraplegia, generalized areflexia and loss of 
general sensitivity function, a diagnosis of brain concussion was made. In $\operatorname{dog} 2$, the transient diffuse cerebellar syndrome associated with normal MRI and CT findings was consistent with cerebellar concussion. In this case, vascular disorders such as cerebellar infarction were ruled out based on a lack of lateralized clinical signs.

It is interesting to note that in dog 1 the biomechanical dynamic of the traumatic event precisely matched what is indicated as cause of cerebral concussion: a vigorous jolting of the head, entirely consistent with the sudden acceleration and/or deceleration of the head that typically induces the syndrome (Shaw, 2002).

These are the first descriptions of non-experimental CNS concussion in dogs.

In several veterinary articles, concussion of the spinal cord is related to the pathophysiology of spinal injury and is described along with laceration and compression as one of the possible effects of intervertebral disc extrusion, fractures, dislocation or subluxation of the vertebral column (Olby, 1999; Ferreira et al., 2002; Kube and Olby, 2008).

In this context, the term "concussion" seems to be used synonymously with spinal "contusion". Differently, human medicine seats the terms "concussion" and "contusion" in two different pathological contexts (Wolmas, 1964; Bedbrook, 1966; Kakulas, 1988; Zwimpfer and Bernstein, 1990; Nakajima et al., 2010; Oyinbo, 2011).

Brain or spinal cord contusion is definable as a blunt trauma to the nervous system ("initial mechanical damage") (Oyinbo, 2011) producing structural alterations, generally followed by persistent or longlasting neurological signs (Gurdjian et al., 1955; Bedbrook, 1966; Bullock et al., 1991; Valsamis, 1994; Van der Naalt, 1997).

Conversely, concussive trauma is characterized by a transient dysfunction of the nervous system without evidence of structural lesions on diagnostic imaging such as radiographs, MRI or CT of the involved anatomical region (Freeman and Wright, 1953; Shaw, 2002).

Spinal cord concussion is supposed to be the result of a delay in the closure of $\mathrm{Na}+$ channels and/or a delay in the opening of $\mathrm{K}+$ channels following depolarization (Zwimpfer and Bernstein, 1990). On the other hand, the mechanism of spinal contusion always involves structural lesions in the nervous system such as hemorrhages and lacerations (Winder et al., 2011).

Furthermore, with regard to the underlying causes, while contusion is caused by traumatic vertebral fractures or luxation, pathological fractures or acute disc extrusions, concussion has a traumatic event as a unique cause.

In conclusion, based on these two first reports of canine spontaneous concussion and on a review of the medical literature, it would also be worthwhile to similarly use the term concussion in veterinary medicine to mean a transient traumatic neurological syndrome without evidence of lesions on diagnostic imaging. In the authors' opinion, it would also be useful to reserve the term contusion for the dynamic consequences of traumatic events on the spinal cord such as fractures, luxation or acute disc extrusions. Finally, considering that the therapeutic approach and prognosis are considerably different in the case of concussive or contusive CNS damage, such clinical and imaging differentiation is of the utmost importance for the management of affected patients.

\section{Conflict of interest}

The authors declare that there is no conflict of interest.

References
Bannister, R.1992. Brain and Bannister's Clinical
Neurology, $7^{\text {th }}$ Edition. Oxford University Press, Oxford.

Bedbrook, B.M. 1966. Pathological Principles In The Management Of Spinal Cord Trauma. Paraplegia 4(1), 43-56.

Blennow, K., Hardy, J., Zetterberg, H. 2012. The neuropathology and neurobiology of traumatic brain injury. Neuron. 76, 886-899.

Bornstein, M.B. 1946. Presence and action of acetylcholine in experimental brain trauma. $\mathbf{J}$. Neurophysiol. 9, 349-366.

Bullock, R., Maxwell, W.L., Graham, D.I., Teasdale, G.M. and Adams, J.H. 1991. Glial swelling following human cerebral contusion: an ultrastructural study. J. Neurol. Neurosurg. Psychiatry 54, 427-434.

Cantu, R.C. 1998. Second-impact syndrome. Clin. Sports Med. 17, 37-44.

Cantu, R.C., Guskiewicz, K. and Register-Mihalik, J.K. 2010. A retrospective clinical analysis of moderate to severe athletic concussions. PM R. 2, 1088-1093.

Cantu, RC. 1969. Transient traumatic cerebellar dysfunction. Report of a syndrome. Int. Surg. 52(5), 392-394.

Denny-Brown, D. and Russell, W.R. 1940. Experimental cerebral concussion. J. Physiol. 99(1), 153.

Ferreira, A.J.A., Correia, J.H.D. and Jaggy, A. 2002. Thoracolumbar disc disease in 71 paraplegic dogs: influence of rate of onset and duration of clinical signs on treatment results. J. Small Anim. Pract. 43, 158-163.

Freeman, L.W. and Wright, T.W. 1953. Experimental observations of concussion and contusion of the spinal cord. Ann. Surg. 137(4), 433-443.

Fumeya, H. and Hideshima, H. 1994. Cerebellar concussion -- three case reports. Neurol. Med. Chir. 34, 612-615. 
Gohil, J.R. and Munshi, S.S. 2006. Post Concussion Ataxia Following Minor Head Injury. Indian Pediatrics 43,829.

Gordon, E. and Ponten, U. 1976. The non-operative treatment of severe head injuries. In Handbook of Clinical Neurology, Vol. 24. Ed. Vinken, P.J., Bruyn, G.W., Braakman, R. (North-Holland, Amsterdam), pp: 599-626.

Gurdjian, E.S., Webster, J.E. and Lissner, H.R. 1955. Observations on the mechanism of brain concussion, contusion, and laceration. Surg. Gynecol. Obstet. 101, 680-690.

Hayes, R.L., Stonnington, H.H., Lyeth, B.G., Dixon, C.E. and Yamamaoto, T. 1986. Metabolic and neurophysiologic sequelae of brain injury: a cholinergic Hypothesis. Cent. Nerv. Syst. Trauma 3(2), 163-173.

Kakulas, A. 1988. The applied neurobiology of human spinal cord injury: a review. Paraplegia 26, 371379.

Kanios, K. 1981. Case of cerebellar concussion. Wiad. Lek. 34(1), 61-62.

Kepski, A. 1983. Clinical aspects and prognosis in cerebellar concussion syndromes. Neurol. Neurochir. Pol. 17(2), 239-240.

Kube, S.A. and Olby, N.J. 2008. Managing acute spinal cord injuries. Compend. Contin. Educ. Vet. 30(9), 496-504.

Label, L.S. 1997. Injuries and Disorders of the Head and Brain. Matthew Bender, New York.

Larrabee, G.J. 1997. Neuropsychological outcome, post concussion symptoms, and forensic considerations in mild closed head trauma. Semin. Clin. Neuropsychiatry 2, 196-206.

Laurent, S., Thibaud, J.L., Hordeaux, J., Reyes-Gomez, E., Delisle, F., Blot, S. and Colle, M.A. 2010. Chronic Traumatic Brain Injury in a Dog. J. Comp. Path. 143, 75-80.

Lorenz, M.D., Coates, J.R. and Kent, M. 2011. Handbook of veterinary neurology. Elsevier Sauders Press, pp: 348-351.

McCrory, P.R. and Berkovic, F.B. 2001. Concussion: the history of clinical and pathophysiological concepts and misconceptions. Neurology. 57, 22832289

McCrory, P., Meeuwisse, W.H., Aubry, M., Cantu, B., Dvorák, J., Echemendia, R.J., Engebretsen, L., Johnston, K., Kutcher, J.S., Raftery, M., Sills, A., Benson, B.W., Davis, G.A., Ellenbogen, R.G., Guskiewicz, K., Herring, S.A., Iverson, G.L., Jordan, B.D., Kissick, J., McCrea, M., McIntosh, A.S., Maddocks, D., Makdissi, M., Purcell, L., Putukian, M., Schneider, K., Tator, C.H. and Turner, M. 2013. Consensus Statement on Concussion in Sport - The $4^{\text {th }}$ International
Conference on Concussion in Sport Held in Zurich, November 2012. Br. J. Sports Med. 47(5), 250-258.

McKee, A.C. 2014. The neuropathology of sport. Acta Neuropathol. 127(1), 29-51.

McKee, A.C., Stein, T.D., Kiernan, P.T. and Alvarez, V.E. 2015. The Neuropathology of Chronic Traumatic Encephalopathy. Brain Pathol. 25(3), 350-364.

Montenigro, P.H., Bernick, C. and Cantu, R.C. 2015a. Clinical Features of Repetitive Traumatic Brain Injury and Chronic Traumatic Encephalopathy. Brain Pathol. 25(3), 304-317.

Montenigro, P.H., Corp, D.T., Stein, T.D., Cantu, R.C. and Stern, R.A. 2015b. Chronic traumatic encephalopathy: historical origins and current perspective. Annu. Rev. Clin. Psychol. 11, 309-330.

Nakajima, Y., Horiuchi, Y., Hiroshi K., Yukawa M., Kuwabara M. and Tsubokawa T. 2010. Distinct time courses of secondary brain damage in the hippocampus following brain concussion and contusion in rats. 2010. Tohoku J. Exp. Med. 221(3), 229-235.

Nesnidal, P., Stulik, J. and Berna, M. 2012. Spinal cord concussion: a retrospective study of twentyfour patients. Acta Chir. Orthop. Traumatol. Cech. 79(2), 150-155.

Olby, N. 1999. Current Concepts in the Management of Acute Spinal Cord Injury. J. Vet. Intern. Med. 13, 399-407.

Ommaya, A.K. and Gennarelli, T.A. 1974. Cerebral concussion and traumatic unconsciousness: correlation of experimental and clinical observations on blunt head injuries. Brain 97, 633654.

Oyinbo, C.A. 2011. Secondary injury mechanisms in traumatic spinal cord injury: a nugget of this multiply cascade. Acta Neurobiol. Exp. 71, 281299.

Petrie, E.C., Cross, D.J., Yarnykh, V.L., Richards, T., Martin, N.M., Pagulayan, K., Hoff, D., Hart, K., Mayer, C., Tarabochia, M., Raskind, M.A., Minoshima, S. and Peskind, E.R. 2014. Neuroimaging, behavioral, and psychological sequelae of repetitive combined blast/impact mild traumatic brain injury in Iraq and Afghanistan war veterans. J. Neurotrauma 31, 425-436.

Platt, S. and Garosi, L. 2012. Small animals neurological emergencies. Manson Publishing Press, pp: 363.

Platt, S.R., Radaelli, S.T. and McDonnell, J.J. 2001. The Prognostic Value of the Modified Glasgow Coma Scale in Head Trauma in Dogs. J. Vet. Intern. Med. 15, 581-584.

Rosenthal, M. 1993. Mild traumatic brain injury syndrome. Ann. Emerg. Med. 22, 1048-1051. 
Sachs, E. 1957. Acetylcholine and serotonin in the spinal fluid. J. Neurosurg. 14, 22-27.

Satz, P.S., Alfano, M.S., Light, R.F., Morgenstern, H.F., Zaucha, K.F., Asarnow, R.F. and Newton, S. 1999. Persistent post-concussive syndrome: A proposed methodology and literature review to determine the effects, if any, of mild head and other bodily injury. J. Clin. Exp. Neuropsychol. 21(5), 620-628.

Scott, W.W. 1940. Physiology of concussion. Arch. Neurol. Psychiatr. 43, 270-283.

Shaw, N.A. 2002. The neurophysiology of concussion. Prog. Neurobiol. 67(4), 281-344.

Snedden, T.R. 2013. Concept analysis of concussion. J. Spec. Pediatr. Nurs. 18(3), 211-220.

Snorf, C. and Lynch, P. 1957. The pathogenesis of cerebral concussion. Q. Bull. Northwest. Univ. Med. Sch. 31(2), 132-135.

Stern, R.A., Daneshvar, D.H., Baugh, C.M., Seichepine, D.R., Montenigro, P.H., Riley, D.O., Fritts, N.G., Stamm, J.M., Robbins, C.A., McHale, L., Simkin, I., Stein, T.D., Alvarez, V.E., Goldstein, L.E., Budson, A.E., Kowall, N.W., Nowinski, C.J., Cantu, R.C. and McKee, A.C. 2013. Clinical presentation of chronic traumatic encephalopathy. Neurology 81(13), 1122-1129.

Stern, R.A., Riley, D.O., Daneshvar, D.H., Nowinski, C.J., Cantu, R.C. and McKee, A.C. 2011. Longterm consequences of repetitive brain trauma: chronic traumatic encephalopathy. PM\&R. 3(10 Suppl. 2), S460-S467.

Torg, J.S., Pavlov, H., Genuario, S.E., Sennett, B., Wisneski, R.J., Robie, B.H. and Jahre, C. 1986.
Neurapraxia of the cervical spinal cord with transient quadriplegia. J. Bone Joint Surg Am. 68(9), 1354-1370.

Tower, D.B. and McEachern, D. 1948. Acetylcholine and neuronal activity in craniocervical trauma. J. Clin. Investig. 27, 558-559.

Trotter, W. 1924. On certain minor injuries of the brain: Being the Annual Oration, Medical Society of London. Br. Med. J. 1(3306), 816-819.

Valsamis, M.P. 1994. Pathology of trauma. Neurosurg. Clin. N. Am. 5(1), 175-183.

Van der Naalt, J. 1997. Concussion and contusion of the brain. Ned Tijdschr. Tandheelkd. 104(11), 418420.

Walker, A.E., Kollros, J.J. and Case, T.J. 1944. The physiological basis of concussion. J. Neurosurg. 1, 103-116.

Winder, M.J., Brett, K. and Hurlbert, R.J. 2011. Spinal cord concussion in a professional ice hockey player. J. Neurosurg. Spine. 14, 677-680.

Windle, W.F., Groat, R.A. and Fox, C.A. 1944. Experimental structural alterations in the brain during and after concussion. Surg. Gynecol. Obstet. 79, 561-572.

Wolmas, L. 1964. The neuropathology of traumatic paraplegia. A critical historical review. Paraplegia. 1, 233-251.

Yeoh, H.K., Lind, C.R.P. and Law, A.J.J. 2006. Acute transient cerebellar dysfunction and stuttering following mild closed head injury. Childs Nerv. Syst. 22, 310-313.

Zwimpfer, T.J. and Bernstein, M. 1990. Spinal cord concussion. J. neurosurg. 72, 894-900. 